\title{
Prior outpatient antibiotic use as predictor for microbial aetiology of community-acquired pneumonia: hospital-based study
}

\author{
Ewoudt MW van de Garde • Henrik Endeman • \\ Remco N van Hemert • G Paul Voorn • \\ Vera HM Deneer • Hubert GM Leufkens • \\ Jules MM van den Bosch • Douwe H Biesma
}

Received: 10 August 2007 / Accepted: 27 October 2007 / Published online: 30 November 2007

(C) The Author(s) 2007

\begin{abstract}
Objective The causative micro-organism in communityacquired pneumonia (CAP) is often difficult to predict. Different studies have examined chronic morbidity and clinical symptoms as predictors for microbial aetiology of pneumonia. The aim of our study was to assess whether prior outpatient antimicrobial treatment is predictive for determining the microbial aetiology of CAP.
\end{abstract}

All authors have read the final version of the manuscript and approved for submission. The manuscript has not been published and is not being considered for publication elsewhere in whole or in part in any language. There is no conflict of interest.

E. M. van de Garde $(\varangle) \cdot$ R. N. van Hemert • V. H. Deneer Department of Clinical Pharmacy, St. Antonius Hospital, P.O. Box 2500, Nieuwegein 3430 EM, The Netherlands e-mail: e.van.de.garde@antoniusmesosgroep.nl

E. M. van de Garde $\cdot$ H. G. Leufkens

Department of Pharmacoepidemiology and Pharmacotherapy, Utrecht Institute for Pharmaceutical Sciences (UIPS),

Utrecht University,

Utrecht, The Netherlands

H. Endeman • D. H. Biesma

Department of Internal Medicine, St. Antonius Hospital,

Nieuwegein, The Netherlands

\section{G. P. Voorn}

Department of Medical Microbiology and Immunology,

St. Antonius Hospital,

Nieuwegein, The Netherlands

J. M. van den Bosch

Department of Pulmonary Medicine, St. Antonius Hospital,

Nieuwegein, The Netherlands
Methods This was a hospital-based prospective observational study including all patients admitted with CAP between 1 October 2004 and 1 August 2006. Microbial investigations included sputum, blood culture, sputum PCR, antigen testing and serology. Exposure to antimicrobial drugs prior to hospital admission was ascertained through community pharmacy dispensing records. Multivariate logistic regression analysis was conducted to assess whether prior outpatient antimicrobial treatment is a predictor of microbial aetiology. Patient demographics, comorbidities and pneumonia severity were considered to be other potential predictors.

Results Overall, 201 patients were included in the study. The microbial aetiology was determined in $64 \%$ of the patients. The five most prevalent pathogens were Streptococcus pneumoniae, Heamophilus influenzae, Legionella spp., Mycoplasma pneumoniae and Influenza virus A+B. Forty-seven of the patients $(23 \%)$ had received initial antimicrobial treatment as outpatients. Multivariate analyses revealed that initial outpatient beta-lactam treatment was associated with a threefold increased chance of finding atypical pathogens and a threefold decreased probability of pneumococcal infection; the corresponding odds ratios were 3.51 (95\% CI 1.25-9.99) and 0.30 (95\% CI 0.10-0.90), respectively. Patients who received macrolides prior to hospitalisation had an increased probability of viral pneumonia.

Conclusion Prior outpatient antimicrobial therapy has a predictive value in the diagnostic workup aimed at identifying the causative pathogen and planning corresponding antimicrobial treatment in patients hospitalised for pneumonia.

Keywords Aetiology · Antibiotics · Diagnosis ·

Outcome assessment $\cdot$ Pneumonia 


\section{Introduction}

Community-acquired pneumonia (CAP) remains a major reason for hospital admission and a common cause of death in developed countries [1,2]. The initial management of patients hospitalised with pneumonia consists mostly of empirical antimicrobial treatment $[3,4]$. Determination of the appropriate antimicrobial treatment is essential as inadequate antimicrobial treatment, generally defined as microbial ineffective therapy against the causative pathogen, can negatively influence patient outcome [5]. Because it is not always possible to identify the causative pathogen, especially during the first days following hospitalisation, many studies have focussed on other parameters suggestive of the causative pathogen. The most frequently studied parameters for this purpose are patient characteristics (age and comorbidities) and clinical signs [6, 7], although nonresponsiveness to prior outpatient antimicrobial treatment has also been suggested as a predictor of the aetiology of pneumonia. However, to date, this latter parameter has not been comprehensively quantified and documented. The aim of the study reported here was to assess whether prior outpatient antimicrobial treatment is a predictor of microbial aetiology in patients admitted to hospital for CAP.

\section{Methods}

The study was conducted in the St. Antonius Hospital, a 600-bed teaching hospital in Nieuwegein, The Netherlands.

\section{Patient population}

This was a prospective observational study of patients with confirmed pneumonia admitted between October 1, 2004 and August 1, 2006. Pneumonia was defined as a new or progressive infiltrate on a chest X-ray plus at least two of the following criteria: cough, sputum production, temperature $>38^{\circ} \mathrm{C}$ or $<35^{\circ} \mathrm{C}$, ausculatory findings consistent with pneumonia, leucocytosis or leucopenia $(>10 \mathrm{~g} / 1,<4 \mathrm{~g} / 1$ or $>10 \%$ rods in leucocyte differentiation), C-reactive protein $>3$ times the upper reference value for normal. Patients who were immune compromised (systemic steroid use at admission (prednisone equivalent $>20 \mathrm{mg}$ /daily for more than 3 days), haematological malignancies and other immunosuppressive therapy) were excluded. The study was approved by the local Medical Ethics Committee, and informed consent was obtained from each patient.

Microbial aetiology workup

At least two blood cultures were performed, and sputum was taken for Gram-staining and culture and subsequently analysed by Taqman real-time PCR for Mycoplasma pneumoniae, Legionella pneumophila and Chlamydophyla psittaci [8]. Pharyngeal samples were taken for viral culture. Urine was sampled for antigen testing on Streptococcus pneumoniae and L.pneumophila (Binax NOW; Binax, Scarborough, ME) $[9,10]$. In addition, serum samples taken on the day of admission and on day 10 were analysed in pairs for detection of a fourfold rise of antibodies to respiratory viruses, Coxiella burnetii, M. pneumoniae, and C. psittaci by complement fixation assay [11]. For each patient, the total workup was completed, and the microbiology department was blinded to data on outpatient antibacterial drug use. When both viruses and bacteria were identified in a patient, the search prevailed for a definite aetiology for the bacteria.

Exposure to antimicrobial therapy

Data on outpatient antimicrobial drug use were acquired through community pharmacy dispensing records that captured all drug exposures the year prior to hospital admission. A patient was considered to be exposed to an antimicrobial drug when a prescription was filled within 14 days prior to hospitalisation. The name, dosage and amount of antimicrobial drug dispensed were also ascertained. The prescribed antimicrobial drug was classified as appropriate or inappropriate in accordance with current Dutch guidelines on the initial treatment of patients with suspected pneumonia [12, 13].

\section{Co-morbidity assessment}

In addition to outpatient antimicrobial drug use, comorbidities and other relevant patient characteristics were identified to address factors related with the aetiology of CAP. Co-morbidities were defined based on the presence of conditions for which the patient was under active medical supervision or was receiving treatment at the time of hospital admission. The co-morbidities evaluated were pulmonary diseases (chronic obstructive pulmonary disease or treated asthma), congestive heart failure, diabetes (both type I and type II), history of stroke and end-stage renal disease (serum creatinine $>150 \mu \mathrm{mol} / \mathrm{l}$ ). The patients were also classified according to the Pneumonia Severity Index (PSI) developed by Fine et al. [14]. This index classifies patients into five categories of predicted mortality risk (with the fifth category being that of highest mortality risk). The outpatient use of oral corticosteroids and gastric acidsuppressing drugs was also ascertained.

Statistical analysis

The SPSS statistical package (ver. 12.0.1 for Windows; SPSS, Chicago, IL) was used for the statistical analyses. 
Continuous data were expressed as the mean $\pm \mathrm{SD}$ or as the median (interquartile range) where appropriate. To study the association between prior outpatient antimicrobial treatment and the aetiology of pneumonia, we applied multivariate logistic regression analyses. The analyses were conducted for overall aetiology and relevant pathogens separately. All baseline characteristics were considered potential predictors. Potential predictors were included in the multivariate model when they were retained after backward stepwise elimination. Significance was set at a $p$ value $<0.05$. The model's performance (goodness-of-fit and discriminative ability) was tested by performing the Hosmer and Lemeshow test and calculating the area under the receiver operator characteristic (ROC) curve.

\section{Results}

In total, 201 patients with pneumonia were included in the study. The mean age of the patients was $63 \pm 17$ years, and 124 patients were male. Three patients (1\%) were admitted from a nursing home. Pneumonia severity as well as comorbid illnesses are summarised in Table 1. The overall median duration of the hospital stay was 10 days [7 -14],

Table 1 Demographics, co-morbidities and pneumonia severity index of 201 patients with community-acquired pneumonia (CAP)

\begin{tabular}{ll}
\hline Characteristic & $n(\%)$ \\
\hline Age (Years) & \\
$<60$ & $74(37)$ \\
$60-69$ & $39(19)$ \\
$70-79$ & $50(25)$ \\
$>80$ & $38(19)$ \\
Gender & \\
Male & $124(62)$ \\
Female & $77(38)$ \\
Co-morbidities & \\
Pulmonary diseases & $71(35)$ \\
Heart failure & $18(9)$ \\
Diabetes & $35(17)$ \\
History of stroke & $17(9)$ \\
End-stage renal disease & $10(5)$ \\
Nursing home resident & $3(1)$ \\
Co-medication & \\
Oral corticosteroids & $58(29)$ \\
Gastric acid suppressing drugs & $61(30)$ \\
Fine score at admission & \\
I & \\
II & $30(15)$ \\
III & $34(17)$ \\
IV & $53(26)$ \\
V & $56(28)$ \\
\hline
\end{tabular}

${ }^{\mathrm{a}}$ Fine et al. [14]
Table 2 Outpatient antibiotics utilization profile prior to hospitalisation for CAP

\begin{tabular}{lll}
\hline Type of antimicrobial drug & Number of users $(\%)^{\mathrm{a}}$ & Appropriate $^{\mathrm{b}}$ \\
\hline Amoxycillin/clavulanic acid & $18(38)$ & Yes \\
Amoxycillin & $12(26)$ & Yes \\
Doxycycline & $7(15)$ & Yes \\
Clarithromycin & $5(11)$ & Yes \\
Co-trimoxazole & $4(8)$ & No \\
Ciprofloxacin & $2(4)$ & No \\
Norfloxacin & $1(2)$ & No \\
Azithromycin & $1(2)$ & Yes \\
\hline
\end{tabular}

${ }^{a}$ Total percentage exceeds $100 \%$ because some patients $(n=3)$ had two prescriptions

${ }^{\mathrm{b}}$ Based on current Dutch guidelines NVALT and SWAB [12, 13]

and 21 patients were admitted to the intensive care ward. During the hospital stay, ten patients died, all due to pneumonia. The overall 28-day mortality rate was $5 \%$. Forty-seven patients $(23 \%)$ had received antimicrobial treatment in the 14-day time-window prior to hospital admission. The antimicrobial drugs dispensed to these patients are summarised in Table 2.

The majority of the patients (79\%) had their prescription filled within 4 days prior to hospital admission, and $85 \%$ of the prescribed antimicrobial drugs complied with current Dutch guidelines $[12,13]$. A microbial aetiology could be determined in $128(64 \%)$ of the patients. Table 3 shows the results of different tests used to determine the aetiological diagnosis of CAP.

Fewer causative pathogens were found in the population hospitalised following prior outpatient antimicrobial treatment than in patients without prior antimicrobial treatment [57 vs. $66 \%$; crude odds ratio (OR) $0.71,95 \%$ confidence interval (CI) 0.36-1.38]. In patients with prior beta-lactam treatment, aetiology in the group comprising atypical bacterial pathogens was more probable (eight of 29 cases, 28\%; crude OR 4.66, 95\% CI 1.73-12.56). The aetiology of $S$. pneumoniae was less prevalent in patients with prior beta-lactam antimicrobial treatment (four of 29 cases, 14\%; crude OR $0.33,95 \%$ CI 0.11-0.99). In multivariate analyses, these associations remained significant (OR $3.51,95 \%$ CI $1.25-9.99$ and OR $0.30,95 \%$ CI $0.10-0.90$, respectively) (Table 4). Additional significant predictors included in these models were heart failure, pulmonary comorbidity, pneumonia severity index and the use of gastric acid suppressing drugs. The goodness-of-fit of both multivariate models was good, with a $p$ value of 0.899 (Hosmer and Lemeshow test) for the model predicting pneumococcal pneumonia and a $p$ value of 0.995 for the model predicting pneumonia of an atypical aetiology. The corresponding areas under the ROC curve were 0.62 and 0.67 , respectively. 
Table 3 Results of of different tests used to determine the aetiology of CAP

\begin{tabular}{|c|c|c|c|c|c|c|}
\hline & Sputum culture & Sputum PCR & Antigen testing & Blood culture & Serology & Viral culture \\
\hline Number of samples & 148 & 78 & 183 & 182 & 130 & 88 \\
\hline Number of positive samples & 78 & 14 & 36 & 19 & 38 & 14 \\
\hline Percentage of positive samples & 53 & 18 & 20 & 10 & 29 & 16 \\
\hline Streptococcus pneumoniae & 33 & - & 30 & 17 & - & - \\
\hline \multicolumn{7}{|l|}{ Gram-negative strain } \\
\hline Heamophilus influenzae & 19 & - & - & 0 & - & - \\
\hline Other & 10 & - & - & 1 & - & - \\
\hline \multicolumn{7}{|l|}{ Atypical } \\
\hline Mycoplasma pneumoniae & - & 7 & - & - & 8 & - \\
\hline Legionella spp. & 1 & 5 & 6 & - & 7 & - \\
\hline Other & - & 2 & - & - & 1 & - \\
\hline Viral & - & - & - & - & 22 & 14 \\
\hline \multicolumn{7}{|l|}{ Other } \\
\hline S. aureus & 6 & - & - & 1 & - & - \\
\hline Gram-positive other & 2 & - & - & 0 & - & - \\
\hline
\end{tabular}

In patients aged $<60$ years without co-morbidities, the aetiology of atypical bacterial pathogens was more prevalent (OR 4.64, 95\% CI 1.72-12.56). Pulmonary comorbidity was associated with the finding of S. pneumoniae and $H$. influenzae as causative pathogens (OR 1.87, 95\% CI 1.00-3.47; OR 3.72, 95\% CI 1.20-11.57, respectively). Patients who received macrolides prior to hospitalisation had an increased probability of viral pneumonia (two of five cases, 40\%) (crude OR 8.67, 95\% CI 1.34-56.23).

\section{Discussion}

The results of our study show that among patients admitted for CAP, a threefold decreased chance of having a $S$. pneumoniae infection and a threefold increased probability of having pneumonia of atypical aetiology were associated with the patient having received an initial beta-lactam treatment as an outpatient. These findings indicate that information on prior outpatient antimicrobial therapy has a predictive value in the diagnostic workup aimed at identifying the causative pathogen and planning the corresponding treatment in patients with pneumonia.

The initial management of patients hospitalised with pneumonia has been under constant study in different settings during the past decades. The choice of antimicrobial treatment, time to first antimicrobial drug administration and route of administration have all appeared to be relevant factors associated with the outcome of pneumonia [15-17]. A knowledge of the predominant microbial patterns in CAP is therefore essential when choosing an essential empirical antimicrobial treatment. Previous studies have found that $S$. pneumoniae, $H$. influenzae, Influenza virus $\mathrm{A}$ and $\mathrm{B}$, Legionella spp. and C. pneumoniae are the most frequent pathogens in CAP [13], which is in accordance with our results. Because $S$. pneumoniae is the most frequently appearing pathogen, the administration of beta-lactam antibiotics is the initial empirical antimicrobial treatment of choice in the treatment guidelines on CAP [3, 12, 13]. Beta-lactam antibiotics, however, do not cover Legionella spp., C. pneumoniae and M. pneumoniae, the so-called atypical pathogens. Therefore, patients with pneumonia of atypical aetiology who are treated with beta-lactam antibiotics as an outpatient will probably not respond to treatment, with the possible consequence being a deterioration of the situation and subsequent hospital admission. Our finding of an increased prevalence of

Table 4 Odds ratios (OR) for aetiology and prior outpatient betalactam treatment in patients admitted to hospital for CAP

\begin{tabular}{llll}
\hline Aetiology & $\begin{array}{l}\text { Prior outpatient beta- } \\
\text { lactam treatment } \\
(n=\text { no. of patients })\end{array}$ & \\
\cline { 2 - 3 } & Yes (\%) & No (\%) & \\
& $29(100)$ & $172(100)$ & \\
\hline Total no. of samples & & & \\
Univariate & $4(14)$ & $56(33)$ & $0.33(0.10-0.99)$ \\
Pneumococcal & $8(28)$ & $13(8)$ & $4.66(1.72-12.56)$ \\
Atypical & $2(7)$ & $14(8)$ & $0.84(0.18-3.89)$ \\
Viral & $2(7)$ & $21(12)$ & $0.53(0.12-2.40)$ \\
Gram-negative strains & $1(3)$ & $7(4)$ & $0.84(0.10-7.11)$ \\
Other & $12(41)$ & $61(36)$ & $1.28(0.58-2.87)$ \\
$\quad$ Unidentified & & & \\
Multivariate & - & - & $0.30(0.10-0.90)^{\mathrm{a}}$ \\
Pneumococcal & - & - & $3.51(1.25-9.99)^{\mathrm{b}}$ \\
Atypical & & &
\end{tabular}

OR, Odds ratio; CI, confidence Interval

${ }^{a}$ Adjusted for heart failure and use of gastric acid suppressing drugs

${ }^{\mathrm{b}}$ Adjusted for pulmonary diseases and pneumonia severity index 
atypical pathogens in patients with prior outpatient betalactam treatment supports such an explanation, but also confirms what has already been suggested in the different guidelines for the management of community-acquired pneumonia in adults $[13,18]$. These guidelines state that following the failure of the initial empirical treatment with beta-lactam antibiotics, the microbiological examination should be reassessed with a view to excluding the less common pathogens, such as atypical pathogens, and that antimicrobial treatment covering atypical pathogens should be considered. Our study supports the choice of antimicrobial treatment covering atypical pathogens (e.g. macrolides) for all patients with CAP who are admitted to hospital after prior treatment with beta-lactam antibiotics. However, whether this protocol will result in improved clinical outcome should be subject to additional study.

The observed reduction in the frequency of S. pneumoniae in patients who received prior outpatient antimicrobial treatment could also be due to a failure to detect the organisms in cultures. This could mask $S$. pneumoniae as the causative pathogen. However, we believe that this explanation is less plausible, especially since we also used antigen testing to identify the causative pathogen [19]. In addition, such a mechanism can not explain the finding of an increased probability of pneumonia caused by atypical pathogens. To the best of our knowledge, our study is the first to specifically document and quantify the failure of initial outpatient antibiotic treatment as a predictor of the microbial aetiology of CAP.

This study was conducted in a single teaching hospital in The Netherlands, but we believe that the conclusions drawn will apply to other settings. First, the percentage of identified aetiology (64\% in this study) is in agreement with that of other studies using similar microbiological techniques [20-22]. Second, our patient characteristics comply to a great extent with a previous nationwide study on prior outpatient antibacterial therapy as a prognostic factor for mortality in patients hospitalised for pneumonia [23]. In that large database study, the percentage of patients hospitalised after initial outpatient antimicrobial treatment was almost identical to that observed in our study (27 vs. $23 \%$, respectively). In addition, age distribution, comorbidities and the antibiotic utilisation profile of the outpatients were very similar as were the median duration of hospital stay and in-hospital mortality. Unfortunately, due to limited numbers, we were unable to study an association between prior outpatient antimicrobial treatment and mortality in our study. A very reassuring finding was that $85 \%$ of all outpatient antibiotic prescriptions complied with national guidelines on the initial treatment of adults with suspected pneumonia. This reduces the possibility that the findings, rather than being associated with antibiotics, might reflect the diagnostic acumen of the physicians who saw the patients in primary care. However, we cannot rule out the possibility that typical signs of infection with atypical pathogens may have been missed by primary care physicians.

As well as finding a relation between prior antimicrobial treatment and aetiology, we also found an association between aetiology and age and pulmonary co-morbidity. Patients aged $<60$ years without co-morbidities were more likely to have an aetiology comprising viral or atypical bacterial pathogens, and pulmonary co-morbidity was independently associated with $S$. pneumoniae and $H$. influenzae as causative pathogens. These findings confirm the results of previous studies on the impact of age and comorbidity on the microbial aetiology of CAP [6]. A limitation of the present study, however, is that we were not able to adjust for smoking habits and alcohol intake of the patients. Previous studies on determinants for pneumonia aetiology found that these factors are significant predictors of pneumococcal infection [6,7]. On the other hand, we do not expect prior antimicrobial therapy and smoking and alcohol intake to coincide in such a way that this would result in a null effect when all the information is available.

In conclusion, among patients admitted for pneumonia, whether or not a patient has received prior antimicrobial therapy as an outpatient provides relevant information in the diagnostic workup, in particular in terms of identifying the causative pathogen and planning the initial treatment at the time of hospital admission. This finding supports a further strengthening of the continuity of care at the interface between the extramural and hospitalised settings.

Open Access This article is distributed under the terms of the Creative Commons Attribution Noncommercial License which permits any noncommercial use, distribution, and reproduction in any medium, provided the original author(s) and source are credited.

\section{References}

1. Fine MJ, Smith MA, Carson CA, Mutha SS, Sankey SS, Weissfeld LA, et al. (1996) Prognosis and outcomes of patients with community-acquired pneumonia. A meta-analysis. JAMA 275(2):134-141

2. Fry AM, Shay DK, Holman RC, Curns AT, Anderson LJ (2005) Trends in Hospitalizations for Pneumonia Among Persons Aged 65 Years or Older in the United States, 1988-2002. JAMA 294 (21):2712-2719

3. BTS Guidelines for the Management of Community Acquired Pneumonia in Adults (2001). Thorax 56[Suppl 4]IV1-64

4. McKean MC (2002) Evidence based medicine: review of BTS guidelines for the management of community acquired pneumonia in adults. J Infect 45(4):213-218 
5. Harbarth S, Nobre V, Pittet D (2007) Does antibiotic selection impact patient outcome? Clin Infect Dis 44(1):87-93

6. Ruiz M, Ewig S, Marcos MA, Martinez JA, Arancibia F, Mensa J, et al. (1999) Etiology of community-acquired pneumonia: impact of age, comorbidity, and severity. Am J Respir Crit Care Med 160 (2):397-405

7. de Roux A, Cavalcanti M, Marcos MA, Garcia E, Ewig S, Mensa $\mathrm{J}$, et al. (2006) Impact of alcohol abuse in the etiology and severity of community-acquired pneumonia. Chest 129(5):12191225

8. Herpers BL, de Jongh BM, van der Zwaluw K, van Hannen EJ (2003) Real-time PCR assay targets the 23S-5S spacer for direct detection and differentiation of Legionella spp. and Legionella pneumophila. J Clin Microbiol 41(10):4815-4816

9. Smith MD, Derrington P, Evans R, Creek M, Morris R, Dance DA, et al. (2003) Rapid diagnosis of bacteremic pneumococcal infections in adults by using the Binax NOW Streptococcus pneumoniae urinary antigen test: a prospective, controlled clinical evaluation. J Clin Microbiol 41(7):2810-2813

10. Helbig JH, Uldum SA, Bernander S, Luck PC, Wewalka G, Abraham B, et al. (2003) Clinical utility of urinary antigen detection for diagnosis of community-acquired, travel-associated, and nosocomial Legionnaires' disease. J Clin Microbiol 41 (2):838-840

11. Taggart EW, Hill HR, Martins TB, Litwin CM (2006) Comparison of complement fixation with two enzyme-linked immunosorbent assays for the detection of antibodies to respiratory viral antigens. Am J Clin Pathol 125(3):460-466

12. Aleva R, Boersma W, Cox A, van Haren E, Scheurs A, Wijnands W, et al. (2001) Guideline diagnosis and treatment of communityacquired pneumonia (in Dutch). In: Dutch Thoracic Society, The Netherlands

13. Schouten JA, Prins JM, Bonten MJ, Degener J, Janknegt RE, Hollander JM, et al. (2005) Revised SWAB guidelines for antimicrobial therapy of community-acquired pneumonia. Neth $\mathbf{J}$ Med 63(8):323-335
14. Fine MJ, Auble TE, Yealy DM, Hanusa BH, Weissfeld LA, Singer DE, et al. (1997) A prediction rule to identify low-risk patients with community-acquired pneumonia. N Engl J Med 336(4):243250

15. Lim WS, van der Eerden MM, Laing R, Boersma WG, Karalus N, Town GI, et al. (2003) Defining community acquired pneumonia severity on presentation to hospital: an international derivation and validation study. Thorax 58(5):377-382

16. Houck PM, Bratzler DW, Nsa W, Ma A, Bartlett JG (2004) Timing of antibiotic administration and outcomes for Medicare patients hospitalized with community-acquired pneumonia. Arch Intern Med 164(6):637-644

17. Oosterheert JJ, Bonten MJ, Schneider MM, Buskens E, Lammers JW, Hustinx WM, et al. (2006) Effectiveness of early switch from intravenous to oral antibiotics in severe community acquired pneumonia: multicentre randomised trial. $\mathrm{Br}$ Med J 333 (7580): 1193

18. Macfarlane JT, Boldy D (2004) 2004 update of BTS pneumonia guidelines: what's new? Thorax 59(5):364-366

19. Korsgaard J, Moller JK, Kilian M (2005) Antibiotic treatment and the diagnosis of Streptococcus pneumoniae in lower respiratory tract infections in adults. Int J Infect Dis 9(5):274-279

20. Woodhead MA, Macfarlane JT, McCracken JS, Rose DH, Finch RG (1987) Prospective study of the aetiology and outcome of pneumonia in the community. Lancet 1(8534):671-674

21. Venkatesan P, Gladman J, Macfarlane JT, Barer D, Berman P, Kinnear W, et al. (1990) A hospital study of community acquired pneumonia in the elderly. Thorax 45(4):254-258

22. Braun JJ, de Graaff CS, de Goey J, Zwinderman AH, Petit PL (2004) Community-acquired pneumonia: pathogens and course in patients admitted to a general hospital. Ned Tijdschr Geneeskd 148(17):836-840

23. van de Garde EM, Souverein PC, van den Bosch JM, Deneer VH, Goettsch WG, Leufkens HG (2006) Prior outpatient antibacterial therapy as prognostic factor for mortality in hospitalized pneumonia patients. Respir Med 100(8):1342-1348 